\title{
Therapeutic drug monitoring in the treatment of active tuberculosis
}

\author{
Aylin Babalik MD¹, Sharyn Mannix MD², Denis Francis BScN², Dick Menzies MD MSc ${ }^{1}$
}

\author{
A Babalik, S Mannix, D Francis, D Menzies. Therapeutic drug \\ monitoring in the treatment of active tuberculosis. Can Respir J \\ $2011 ; 18(4): 225-229$.
}

BACKGROUND: Therapeutic drug monitoring (TDM) is used to optimize dosing that maximizes therapeutic benefit while minimizing toxicity. In the treatment of active tuberculosis (TB), TDM is not routine, yet low levels of anti-TB drugs can be associated with poorer treatment outcomes. METHODS: In a retrospective case control study, patients with active TB in whom TDM was performed were considered cases and compared with controls who did not undergo TDM, and matched according to year of diagnosis and the results of direct smear microscopy. Medical records were reviewed to abstract demographic, clinical, radiographic and microbiological data including time until smear and culture conversion.

RESULTS: In total, 20 patients were identified in whom TDM was performed, of whom $17(87 \%)$ had at least one low drug concentration. Overall, 27 of $45(60 \%)$ initial drug concentrations were low and resulted in an increased drug dosage. Low drug levels were found in 13 of $15(87 \%)$ isoniazid, four of five (80\%) rifabutin and eight of $12(67 \%)$ rifampin measurements, but in only two of $13(15 \%)$ pyrazinamide measurements. Within cases only, the 17 patients with low serum drug levels were significantly more likely to have comorbid illnesses, be smear positive, have lower serum albumin levels and had nonsignificantly longer time to culture conversion, compared with the three cases in whom all drug levels were within therapeutic ranges.

CONCLUSIONS: TB drug levels were frequently below clinically acceptable levels in patients with active TB, particularly in those with HIV infection or other comorbidities. TDM is potentially useful for the treatment of active $\mathrm{TB}$, but is currently underused.

Key Words: Antituberculosis drugs; Pharmacokinetics; Pharmacology; Therapeutic drug monitoring; Tuberculosis treatment

$\mathrm{T}$ he WHO estimated that 9.2 million persons worldwide developed active tuberculosis (TB) in 2008. In the vast majority of countries, patients with newly diagnosed, active TB received standardized treatment with a six-month regimen containing rifampin throughout, which is recommended for low- and middle-income countries (1), as well as high-income countries such as Canada (2) and the United States (3). In adults with drug-sensitive TB, this regimen, with weightadjusted dosing, has shown high efficacy in a large number of randomized trials (4).

Therapeutic drug monitoring (TDM) has been used in the treatment of a variety of conditions to ensure optimal dosing that maximizes therapeutic benefit while minimizing toxicity (5). In the treatment of active TB, TDM is not part of routine practice, which reflects both the efficacy of the regimen and the high cost and complexity of the tests. TDM for TB drugs is performed in a handful of laboratories in the United States and none in Canada. However, several studies have found that low levels of isoniazid (INH) and rifampin are common in patients treated for active TB $(6,7)$, and that low levels can be associated with slow response to therapy (8), increased risk of relapse (9) and acquired drug resistance (10).

Over the past five years, our centre has used TDM in patients treated for active TB, although only for selected patients because the

\section{La pharmacovigilance thérapeutique dans le traitement de la tuberculose active}

HISTORIQUE : La pharmacovigilance thérapeutique (PVT) permet d'optimiser la posologie afin d'en tirer le maximum d'avantages thérapeutiques tout en en réduisant la toxicité au minimum. Dans le traitement de la tuberculose (TB) active, la PVT n'est pas systématique, mais de faibles taux de médicaments antituberculeux peuvent s'associer à une moins bonne issue des traitements.

MÉTHODOLOGIE : Dans le cadre d'une étude cas-témoins rétrospective, les patients atteints de TB active chez qui on avait procédé à la PVT étaient considérés comme des cas et comparés à des sujets témoins qui n'avaient pas subi la PVT, puis ont été jumelés selon l'année du diagnostic et les résultats d'un frottis direct. Les chercheurs ont analysé les dossiers médicaux afin d'en extraire les données démographiques, cliniques, radiographiques et microbiologiques, y compris le délai jusqu'au frottis et la conversion de la culture.

RÉSULTATS : Au total, la PVT avait été exécutée chez 20 patients, dont 17 (87\%) présentaient au moins une faible concentration de médicaments. Dans l'ensemble, 27 des 45 (60\%) concentrations initiales de médicaments étaient faibles et ont suscité une augmentation de la posologie. Les chercheurs ont constaté de faibles taux d'isoniazide dans 13 cas sur 15 (87\%), de rifabutine dans quatre cas sur cinq ( $80 \%)$ et de rifampine dans huit cas sur 12 (67\%), mais de pyrazinamide seulement dans deux cas sur 13 (15\%). Dans les cas seulement, les 17 patients présentant un faible taux sérique de médicaments étaient considérablement plus susceptibles d'avoir des maladies positives, un frottis positif, un taux d'albumine sérique faible et une période plus longue, mais non significative, jusqu'à la conversion de la culture que les trois cas dans lesquels les taux de médicaments se situaient dans la plage thérapeutique.

CONCLUSIONS : Les taux de médicaments contre la TB se situaient souvent sous les taux cliniquement acceptables chez les patients atteints de TB active, notamment ceux atteints d'une infection par le VIH et d'autres comorbidités. La PVT pourrait être utile pour traiter la TB active, mais elle est actuellement sous-utilisée.sous les taux cliniquement acceptables chez les patients atteints de TB active, notamment ceux atteints d'une infection par le VIH et d'autres comorbidités. La PVT pourrait être utile pour traiter la TB active, mais elle est actuellement sous-utilisée.

service was costly and difficult to access. We reviewed our results over the past five years to examine the frequency of finding low drug levels, the dosing adjustments necessary to achieve adequate levels and factors associated with low drug levels.

\section{Data gathering}

\section{METHODS}

A retrospective case control study was conducted. Medical records of all patients treated for active TB between 2005 and the present were reviewed to identify those in whom TDM was performed. All patients with confirmed pulmonary active TB treated for culture conversion in whom serum concentrations of at least one anti-TB drug were measured, were considered cases. For each case, a control patient with active culture-positive pulmonary TB in whom drug levels were not measured was selected. Controls were matched to cases according to the results of direct smear microscopy (ie, acid-fast bacillus [AFB] smear positive or negative) and year of diagnosis - the latter because of potential changes in the management of these patients over time.

At the centre, patients whose initial sputum AFB smears are positive have sputum samples examined once weekly until at least two consecutive sputum samples are AFB smear negative. In all patients, sputum is sent for AFB smear testing and culture after two months of therapy.

${ }^{1}$ Respiratory Epidemiology and Clinical Research Unit, Montreal Chest Institute, McGill University; ${ }^{2}$ Montreal Chest Institute, Montreal, Quebec Correspondence: Dr Dick Menzies, Respiratory Epidemiology and Clinical Research Unit, Montreal Chest Institute, 3650 St Urbain Street,

Room K1.24, Montreal, Quebec H2X 2P4. Telephone 514-934-1934 ext 32128, fax 514-843-2083, e-mail dick.menzies@mcgill.ca 
TABLE 1

Comparison of clinical features between cases and controls

\begin{tabular}{|c|c|c|c|}
\hline Variable & Controls* $(n=20)$ & Cases* $^{*}(n=20)$ & $\mathbf{P}$ \\
\hline Male sex, n (\%) & $9(45)$ & $13(65)$ & 0.20 \\
\hline Age, years & $34.3 \pm 10.3$ & $42.4 \pm 14.1$ & 0.04 \\
\hline Weight, kg & $65.9 \pm 11.5$ & $61.4 \pm 14.0$ & 0.27 \\
\hline HIV infected, $\mathrm{n}(\%)^{\dagger}$ & $0(0)$ & $8(40)$ & 0.0016 \\
\hline Bilateral lesion on chest radiography, $\mathrm{n}(\%)$ & $0(0)$ & $7(35)$ & 0.0036 \\
\hline Cavity on chest radiography, n (\%) & $4(20)$ & $7(35)$ & 0.29 \\
\hline Albumin, $g / L$ & $39.2 \pm 3.9$ & $31.7 \pm 6.6$ & 0.0004 \\
\hline White blood cell count, $\times 10^{9} / \mathrm{L}$ & $6.9 \pm 2.7$ & $10.9 \pm 10.7$ & 0.12 \\
\hline Hemoglobin, g/L & $13.3 \pm 1.3$ & $12.5 \pm 1.3$ & 0.04 \\
\hline Weeks to smear conversion & $4.7 \pm 2.1$ & $7.9 \pm 7.3$ & 0.16 \\
\hline Weeks to culture conversion & $6.4 \pm 3.1$ & $7.05 \pm 5.8$ & 0.68 \\
\hline Total duration of antituberculosis therapy, weeks & $26.5 \pm 4.2$ & $33.4 \pm 8.1$ & 0.005 \\
\hline
\end{tabular}

Data presented as mean \pm SD unless otherwise indicated. *Cases were defined as patients who underwent therapeutic drug monitoring for at least one antituberculosis drug while on treatment for active tuberculosis. All cases and controls had culture-confirmed active pulmonary tuberculosis; ${ }^{\dagger}$ Controls were matched on sputum acid-fast bacillus smear, as well as year of diagnosis; ${ }^{\ddagger}$ All cases and controls were tested for HIV infection

The medical records of each patient were reviewed to abstract demographic and clinical data, body weight, radiographic findings, treatment information including dosages, and microbiological findings including AFB smear, culture, drug sensitivity tests, and time until smear and culture conversion. Newly diagnosed patients with drugsensitive organisms received INH $5 \mathrm{mg} / \mathrm{kg}$ up to a maximum of $300 \mathrm{mg}$ daily, rifampin $10 \mathrm{mg} / \mathrm{kg}$ up to $600 \mathrm{mg}$ daily, pyrazinamide (PZA) $20 \mathrm{mg} / \mathrm{kg}$ (with no maximum) and ethambutol $15 \mathrm{mg} / \mathrm{kg}$ (no maximum). Rifabutin dosing was based on published guidelines for treatment of active TB in HIV-coinfected patients (11,12). For the majority of patients, therapy was given daily and was self-administered. Fixed-dose combinations were not used.

\section{TDM}

Patients were selected for TDM because their treating physician was concerned about a slow clinical response to therapy, which was judged on the basis of weekly AFB smear tests in patients whose initial sputum smears were AFB positive. In all patients with culture-confirmed active pulmonary $\mathrm{TB}$, sputum was sent for AFB smear testing and TB culture after two months. Additional patients underwent TDM if they were undergoing concomitant treatment or had comorbid illnesses (such as treatment for HIV infection) that led to uncertainty about correct drug dosing. Patients underwent TDM after at least two weeks of therapy. On the morning that levels were drawn, patients ingested their usual doses of all medications under direct observation. Venous blood was drawn $2 \mathrm{~h}$ later, the serum was separated, and samples were then frozen and shipped on dry ice to the mycobacteriology laboratory at the National Jewish Hospital (Colorado, USA).

Drug concentrations were compared with published reference ranges from studies in human volunteers $(5,13)$. If drug levels were low, the treating physician would adjust the doses upward; the change in dosage was at the physician's discretion (ie, without a fixed protocol or algorithm). After a minimum of two weeks of treatment with the new TB drug dosages, serum drug levels were re-assessed to ascertain whether they were now in the therapeutic range. If drug levels remained suboptimal, the drug dosages were adjusted a third time. In the first two to three years of TDM monitoring, there were considerable local administrative problems related to payment for these assays, which were not available in Canada. As a result, for many patients, there were lengthy delays of two to three months between sample acquisition and receiving the results. Therefore, in some patients, dosages were not adjusted because the results were received too late, while in several others, the dose adjustments were made, but the treating physician judged that there was not sufficient time of therapy remaining to warrant taking repeat measurements (because they assumed the same delay would occur).

Data analysis

Differences between cases and controls, between HIV-positive and HIV-negative cases, or between cases with low or normal drug levels were tested for significance using $\chi^{2}$ tests for categorical variables and two-sided independent $t$ tests for continuous variables (14). All analyses were conducted using SAS version 9.2 (SAS Institute, USA).

\section{RESULTS}

In total, between 2005 and the present, 20 patients were identified in whom TDM was performed at least once. This represented $7 \%$ of the total of 274 patients treated over the same interval. As shown in Table 1, the mean age of these 20 patients was 42.4 years, with $65 \%$ being men. The most significant difference between the 20 cases and their controls was that eight of the cases and none of the controls were HIV infected $(\mathrm{P}=0.007)$. In addition, cases were significantly more likely to have comorbid illnesses other than HIV, were somewhat older, had more severe radiographic disease with bilateral lesions on chest $\mathrm{x}$-ray, and had lower serum albumin and hemoglobin levels. Of the 20 cases, 16 had drug-sensitive TB, one had a mono INH-resistant strain of TB and three had multidrug-resistant TB. Results of the measurements of the second-line drugs are not presented because there were only one or two measurements per drug.

Among these 20 patients, 45 different drug levels were measured initially, yielding an average of 2.25 drug levels measured per patient. In total, $17(87 \%)$ of the cases had at least one low drug level. Twentyseven $(60 \%)$ of these measurements were low and resulted in an increased drug dosage, following which drug levels were remeasured in 18. In the other nine instances, drug levels were not remeasured because of local administrative delays and the difficulties mentioned above. The total cost (not including costs associated with local handling, but including shipping and laboratory charges for the assays after conversion to $\mathrm{CAD} \$$ ) was $\$ 9200$ (ie, approximately $\$ 460$ per patient).

As shown in Table 2, low levels of INH were the most frequently seen (13 of 15 [87\%] patients), while rifabutin levels were low in four of five $(80 \%)$ patients, and rifampin levels were low in eight of $12(67 \%)$ patients. Of note, only two of 13 (15\%) PZA measurements were below the therapeutic range. The biggest increase in dosage was for rifabutin; on average, the dosage of this drug was almost tripled and subsequent measurements averaged low normal levels. Increases in INH dose were also substantial, averaging $60 \%$ of the premeasurement dose, which resulted in a $120 \%$ increase in drug levels. 


\begin{tabular}{|c|c|c|c|c|}
\hline & Isoniazid & Rifampin & Rifabutin & Pyrazinamide \\
\hline Range of clinically acceptable values, $\mu \mathrm{g} / \mathrm{mL}$ & $3-6$ & $8-24$ & $0.3-0.9$ & $20-60$ \\
\hline \multicolumn{5}{|l|}{ Initial measurements } \\
\hline Number measured, $\mathrm{n}$ & 15 & 12 & 5 & 13 \\
\hline Initial dose, mg & $286 \pm 35$ & $575 \pm 58$ & $60 \pm 9$ & $1384 \pm 194$ \\
\hline Initial dose, $\mathrm{mg} / \mathrm{kg}$ & $5.0 \pm 1.0$ & $10.2 \pm 1.3$ & $0.8 \pm 0.3$ & $23.9 \pm 3.6$ \\
\hline Initial drug level, $\mu \mathrm{g} / \mathrm{mL}$ & $2.0 \pm 1.3$ & $9.1 \pm 4$ & $0.2 \pm 0.1$ & $32.9 \pm 11.3$ \\
\hline Increase in dose (only if changed), $\%$ & 66 & 52 & 187 & 33 \\
\hline Second dose (only if changed), $\mathrm{mg} / \mathrm{kg}$ & $8.1 \pm 1.9$ & $10.6 \pm 6.1$ & $2.5 \pm 0.9$ & $25.5 \pm 12.6$ \\
\hline \multicolumn{5}{|l|}{ Repeat measurements* } \\
\hline Number remeasured, $\mathrm{n}$ & 9 & 3 & 4 & 2 \\
\hline Initial dose (before measurement), mg/kg & $5.1 \pm 0.9$ & $10 \pm 1$ & $0.8 \pm 0.3$ & $21.2 \pm 6.5$ \\
\hline Initial drug level, $\mu \mathrm{g} / \mathrm{mL}$ & $1.5 \pm 0.9$ & $6.9 \pm 2.5$ & $0.2 \pm 0.1$ & $24.6 \pm 10.3$ \\
\hline
\end{tabular}

Data presented as mean $\pm S D$ unless otherwise indicated. *Patients underwent remeasurement of drug levels only if tuberculosis drug dosages were adjusted based on initial drug level measurements

TABLE 3

Factors associated with initially low tuberculosis drug levels (any low level) among patients who had levels measured (cases)

\begin{tabular}{|c|c|c|c|}
\hline Variable & Any low level $(n=17)$ & Levels all normal $(n=3)$ & $\mathbf{P}$ \\
\hline Male sex, n (\%) & $13(76)$ & 0 & 0.01 \\
\hline Weight, kg & $61.8 \pm 14.4$ & $59.1 \pm 13.9$ & 0.78 \\
\hline Presence of comorbid illnesses, $\mathrm{n}(\%)$ & $13(76)$ & $0(0)$ & 0.01 \\
\hline HIV infected, n (\%) & $8(47)$ & $0(0)$ & 0.12 \\
\hline Bilateral lesion on chest radiography, n (\%) & $5(29)$ & $2(67)$ & 0.21 \\
\hline Initially smear positive, n (\%) & $11(64)$ & $0(0)$ & 0.04 \\
\hline Albumin, $g / L$ & $30.5 \pm 6.6$ & $36.3 \pm 4.5$ & 0.17 \\
\hline White blood cell count, $\times 10^{9} / \mathrm{L}$ & $11.5 \pm 11.4$ & $7.6 \pm 4.2$ & 0.57 \\
\hline Hemoglobin, g/L & 12.3 & $13.4 \pm 1.5$ & 0.20 \\
\hline Weeks to smear conversion & $7.9 \pm 7.3$ & - & - \\
\hline Weeks to culture conversion & $7.8 \pm 5.9$ & $3.3 \pm 4$ & 0.23 \\
\hline
\end{tabular}

Data presented as mean \pm SD unless otherwise indicated

The 17 cases with low serum drug levels were more likely to have comorbid illnesses, be smear positive and have lower serum albumin levels compared with the three cases in whom all drug levels were within therapeutic ranges (Table 3 ). The comorbid illnesses among cases were HIV $(n=8)$, liver disease $(n=2)$, chronic renal insufficiency $(\mathrm{n}=2)$ and diabetes $(\mathrm{n}=1)$. Mean time to culture conversion was 7.8 weeks in those with low levels compared with 3.3 weeks in those with adequate levels - a nonsignificant difference. The time to treatment completion was longer in cases than in controls, but was not different in cases with low or normal levels. Low INH levels were associated with comorbid illnesses (Table 4). Low INH levels were nonsignificantly associated with HIV infection and a slightly longer time to smear conversion. Low rifampin or rifabutin levels were associated with lower dose per $\mathrm{kg}$ and with a trend toward longer time to smear or culture conversion. PZA levels were low in only two patients; these were associated with a longer time to smear and culture conversion (Table 4).

As shown in Table 5, when the eight HIV-infected cases were compared with the remaining cases, they had a lower average dose (in $\mathrm{mg} / \mathrm{kg}$ ), a slightly longer time to conversion and lower INH levels; these differences, however, were not significant. Rifabutin concentrations in four of the five HIV-infected individuals who received this drug were low and required substantial dosage adjustment, as shown in Table 2.

\section{DISCUSSION}

In the present retrospective case control study, 20 patients were identified in whom TDM was performed, with 17 having at least one low drug level. Overall, $60 \%$ of all drug levels were below the normal range and required dose adjustment. The most frequent low levels were of INH, rifampin and rifabutin. Among TB patients who underwent TDM, low levels were associated with comorbid illnesses such as HIV infection and more extensive disease.

These findings are of interest to clinicians caring for TB patients, but must be tempered by several important limitations of the present study. First, patients were highly selected because they represented less than $10 \%$ of all TB patients treated during the study interval. Second, 
TABLE 4

Factors associated with initially low levels of specific tuberculosis drugs among patients who had levels measured (cases)

\begin{tabular}{|c|c|c|c|c|c|c|c|c|c|}
\hline \multirow[b]{2}{*}{ Variable } & \multicolumn{3}{|c|}{ Isoniazid levels } & \multicolumn{3}{|c|}{ Rifampin or rifabutin levels } & \multicolumn{3}{|c|}{ Pyrazinamide levels } \\
\hline & $\begin{array}{c}\text { Low } \\
(n=13)^{*}\end{array}$ & $\begin{array}{c}\text { Normal } \\
(n=2)\end{array}$ & $\mathbf{P}$ & $\begin{array}{c}\text { Low } \\
(n=12)^{\star}\end{array}$ & $\begin{array}{c}\text { Normal } \\
(n=5)\end{array}$ & $\mathbf{P}$ & $\begin{array}{c}\text { Low } \\
(n=2)^{\star}\end{array}$ & $\begin{array}{l}\text { Normal } \\
(n=11)\end{array}$ & $\mathbf{P}$ \\
\hline Male sex, n (\%) & $10(77)$ & $0(0)$ & 0.03 & $8(66.7)$ & $5(100)$ & 0.14 & $2(100)$ & $7(64)$ & 0.31 \\
\hline Age, years & $44.3 \pm 15.1$ & $28 \pm 2.8$ & 0.16 & $46.7 \pm 15.3$ & $37.6 \pm 10.5$ & 0.24 & $40.5 \pm 0.7$ & $36.8 \pm 10$ & 0.62 \\
\hline Weight, kg & $60.3 \pm 14.1$ & $53.3 \pm 0.4$ & 0.50 & $64.9 \pm 15.5$ & $54.1 \pm 7.6$ & 0.16 & $57.5 \pm 0.7$ & $59.1 \pm 13.3$ & 0.87 \\
\hline History of previous treatment, n (\%) & $4(31)$ & $0(0)$ & 0.35 & $3(25)$ & $2(40)$ & 0.53 & $1(50)$ & $5(45)$ & 0.91 \\
\hline Presence of comorbid illnesses, n (\%) & $10(77)$ & $0(0)$ & 0.03 & $9(75)$ & $4(80)$ & 0.82 & $2(100)$ & $6(54)$ & 0.22 \\
\hline HIV infected, n (\%) & $5(38)$ & $0(0)$ & 0.28 & $6(50)$ & $2(40)$ & 0.70 & $2(100)$ & $3(27)$ & 0.05 \\
\hline Bilateral lesion on chest radiography, n (\%) & $4(31)$ & $1(50)$ & 0.59 & $1(8.3)$ & $4(80)$ & 0.003 & $1(50)$ & $6(55)$ & 0.90 \\
\hline Cavity on chest radiography, $\mathrm{n}(\%)$ & $6(46)$ & $0(0)$ & 0.21 & $5(41.6)$ & $2(40)$ & 0.94 & $2(100)$ & $3(27)$ & 0.05 \\
\hline Initially smear positive, n (\%) & $9(69)$ & $1(50)$ & 0.60 & $7(58)$ & $4(80)$ & 0.39 & $2(100)$ & $6(54)$ & 0.22 \\
\hline Weeks to smear conversion & $6.11 \pm 3.1$ & 4.0 & 0.54 & $9.4 \pm 8.8$ & $5.3 \pm 2.1$ & 0.42 & $18 \pm 14.1$ & $5.3 \pm 2.2$ & 0.04 \\
\hline Weeks to culture conversion & $6.7 \pm 4.1$ & $6.0 \pm 2.8$ & 0.81 & $8.9 \pm 7.04$ & $5.6 \pm 1.5$ & 0.32 & $15 \pm 14.2$ & $4.1 \pm 2.1$ & 0.01 \\
\hline
\end{tabular}

Data presented as mean \pm SD unless otherwise indicated. *Levels were low and dose was increased by the treating physician

\section{TABLE 5}

Comparison of clinical features and initial tuberculosis drug levels according to HIV status among patients who had drug levels measured (cases)

\begin{tabular}{|c|c|c|c|}
\hline \multirow[b]{2}{*}{ Variable Name } & \multicolumn{2}{|c|}{ HIV status } & \multirow[b]{2}{*}{$\mathbf{P}$} \\
\hline & $\begin{array}{c}\text { Positive } \\
(n=8)\end{array}$ & $\begin{array}{c}\text { Negative } \\
(n=12)\end{array}$ & \\
\hline Male sex, n (\%) & $7(87)$ & $6(50)$ & 0.09 \\
\hline Age, years & $44.1 \pm 11.8$ & $41.1 \pm 11.8$ & 0.67 \\
\hline Weight, kg & $68 \pm 16$ & $57 \pm 11$ & 0.08 \\
\hline History of previous treatment, $\mathrm{n}(\%)$ & $4(50)$ & $3(25)$ & 0.25 \\
\hline $\begin{array}{l}\text { Bilateral lesion on chest radiography, } \\
\mathrm{n}(\%)\end{array}$ & $5(62)$ & $4(33)$ & 0.84 \\
\hline Cavity on chest radiography, $\mathrm{n}(\%)$ & $4(50)$ & $3(25)$ & 0.25 \\
\hline Initially smear positive, n (\%) & $4(50)$ & $7(58)$ & 0.71 \\
\hline Weeks to smear conversion & $13.3 \pm 10.2$ & $4.9 \pm 2.3$ & 0.06 \\
\hline Weeks to culture conversion & $8.8 \pm 7.1$ & $5.6 \pm 4.4$ & 0.25 \\
\hline Albumin, $g / L$ & $30 \pm 6.6$ & $33.3 \pm 7.4$ & 0.39 \\
\hline White blood cell count, $\times 10^{9} / \mathrm{L}$ & $8.6 \pm 3.7$ & $14.4 \pm 16.3$ & 0.24 \\
\hline Hemoglobin, g/L & $12.5 \pm 1.5$ & $12.4 \pm 1.5$ & 0.99 \\
\hline \multicolumn{4}{|l|}{ Isoniazid } \\
\hline Patients measured, $\mathrm{n}$ & 5 & 10 & \\
\hline Initial dose, $\mathrm{mg} / \mathrm{kg}$ & $4.39 \pm 0.75$ & $5.26 \pm 0.68$ & \\
\hline Initial drug level, $\mu \mathrm{g} / \mathrm{mL}$ & $1.48 \pm 0.81$ & $2.30 \pm 1.37$ & 0.27 \\
\hline Levels low and dose increased, n (\%) & $5(100)$ & $8(80)$ & \\
\hline \multicolumn{4}{|l|}{ Rifampin* } \\
\hline Patients measured, $\mathrm{n}$ & $3^{*}$ & 9 & \\
\hline Initial dose, $\mathrm{mg} / \mathrm{kg}$ & $10.01 \pm 0.74$ & $10.25 \pm 1.46$ & \\
\hline Initial drug level, $\mu \mathrm{g} / \mathrm{mL}$ & $10.00 \pm 5.55$ & $8.73 \pm 3.88$ & 0.79 \\
\hline Levels low and dose increased, n (\%) & $2(67)$ & $6(67)$ & \\
\hline \multicolumn{4}{|l|}{ Pyrazinamide } \\
\hline Patients measured, $\mathrm{n}$ & 5 & 8 & \\
\hline Initial dose, $\mathrm{mg} / \mathrm{kg}$ & $18.1 \pm 7.1$ & $15.8 \pm 6.2$ & \\
\hline Initial drug level, $\mu \mathrm{g} / \mathrm{mL}$ & $28.4 \pm 8.9$ & $35.9 \pm 12.1$ & 0.26 \\
\hline Levels low and dose increased, $\mathrm{n}(\%)$ & $2(40)$ & $0(0)$ & \\
\hline
\end{tabular}

Data presented as mean $\pm S D$ unless otherwise indicated. ${ }^{*}$ Five HIV-positive individuals had rifabutin levels measured but no HIV-negative individuals were treated with rifabutin. Therefore, rifabutin results are only shown in Table 2. However, all rifabutin levels were low and required dosage increase

serum samples for measurements of all TB drugs were made at the same time (ie, $2 \mathrm{~h}$ after TB drug ingestion) to minimize patient discomfort and inconvenience. This could have resulted in an underestimate of peak concentrations in patients with rapid or delayed absorption of INH (ie, peak levels have been described in some patients after $1 \mathrm{~h}$, or up to $6 \mathrm{~h}$ in others [5]). It is also possible that peak rifabutin concentrations were underestimated with a $2 \mathrm{~h}$ measurement because peak concentrations are usually achieved after $3 \mathrm{~h}$ to $4 \mathrm{~h}$ (5). However, rifabutin levels were so low that it seems implausible that this was entirely due to drawing samples early. Third, drug levels were not measured in controls; hence, some of them may have had abnormally low levels. This would result in misclassification and a tendency to reduce differences between cases and controls. Fourth, repeat measurements were not always performed, even in patients whose dosages were adjusted. Hence, the estimation of dose adjustment necessary to achieve therapeutic drug levels was incomplete. The small number of patients reduced our power to detect significant associations. Finally, we could not judge whether clinical outcomes were improved by the adjustment of drug doses; ultimately, all patients were cured; however, the duration of therapy was significantly longer for cases.

We did not match patients with respect to speed of response because of concern that it could have resulted in over matching, thereby reducing our ability to detect differences in associated clinical characteristics. In addition, because all patients who remained smear positive after two months of therapy underwent TDM, finding matched controls would have required using historical controls, with the attendant potential confounding changes in general management of these patients.

Nevertheless, an important clinical implication is the common finding of low levels of INH and rifamycins (rifampin or rifabutin), although levels of PZA were usually normal. While this may be partially explained by the high degree of patient selection, it is in keeping with two studies of consecutive TB patients conducted in Botswana (6,15): $30 \%$ to $37 \%$ had low levels of INH, $78 \%$ to $84 \%$ had low levels of rifampin, but only $1 \%$ to $5 \%$ had low levels of PZA. In an Australian study (16), $48 \%$ of INH levels and $46 \%$ of rifampin levels were below the normal range, while in Indonesia, $70 \%$ of 62 TB patients had low rifampin concentrations (7). Similar to our findings, low drug levels in other studies were associated with HIV infection $(15,17,18)$ and low serum albumin levels (6), suggesting chronic malnutrition. Other reliable markers that would indicate which patients are likely to have low drug levels have not been identified.

In our study, patients with low drug levels had longer time to culture conversion, as was reported by others (8). However, final treatment outcomes were not affected because doses were adjusted and therapy was often prolonged; ultimately, all patients were cured. However, there is some published evidence that low drug levels are associated with poorer overall treatment outcome (15), failure and relapse (9), and acquired resistance (10). This suggests that the cost, which averaged $\$ 460$ per patient in our study, and US $\$ 619$ in another recent study (18), may be justified given that total costs related to each case of active TB in Canada in 2004 averaged \$47,290 (19). 
One important implication is whether current dosing of rifampin is adequate; others have suggested that current dosing may be suboptimal, and trials are needed to evaluate the efficacy of higher dose rifampin (20). A second important implication was our finding that four of the five patients on rifabutin had very low levels. Initial doses were calculated using available published guidelines for rifabutin dosing in patients on antiretroviral therapy $(11,12)$. Although inferences are limited by the small number of patients, our finding underscores the current uncertainty for rifabutin dosing in patients on antiretroviral therapy $(11,12)$, and emphasize the importance of TDM in HIV-infected patients on antiretroviral therapy soon after they begin TB therapy.

In summary, low drug levels were frequent among patients on therapy for active TB, particularly among patients with HIV infection or other comorbidities. Although low drug levels are difficult to predict, there is evidence - from the current and other studies - that low levels are clinically important. There is no doubt that large-scale prospective cohort studies or randomized trials would provide better evidence regarding the clinical relevance and optimal concentrations of TB drug levels. Nevertheless, results of the present study and others suggest that achievement of the target serum levels for each TB drug listed in Table 2 is an important objective. In our experience, doses of INH and rifampin had to be increased by $50 \%$ or more to achieve adequate levels in most patients. We found, as have others $(15,17,18)$, that the rifabutin doses required to achieve adequate serum levels were very difficult to predict in HIV-coinfected patients on antiretroviral

\section{REFERENCES}

1. World Health Organization. Treatment of Tuberculosis Guidelines, 4th edn. Geneva: World Health Organization; 2009. Report No.: WHO/HTM/TB/2009.420.

2. Long RL, Ellis E. Canadian Tuberculosis Standards, 6th edn. Toronto: Canadian Lung Association, Public Health Agency of Canada, Tuberculosis Prevention and Control, 2007.

3. American Thoracic Society, Infectious Diseases Society of America, Centres for Disease Control. Treatment of tuberculosis. Am J Respir Crit Care Med 2003;167:603-62.

4. Menzies D, Benedetti A, Paydar A, et al. Effect of duration and intermittency of rifampin on tuberculosis treatment outcomes: A systematic review and meta-analysis. PLOS Med 2009;6:e1000146.

5. Peloquin $\mathrm{C}$. Therapeutic drug monitoring in the treatment of tuberculosis. Drugs 2002;62:2169-83.

6. Tappero J, Bradford W, Agerton T, et al. Serum concentrations of antimycobacterial drugs in patients with pulmonary tuberculosis in Botswana. Clin Infect Dis 2005;41:461-9.

7. van Crevel R, Alisjahbana B, de Lange WCM, et al. Low plasma concentrations of rifampicin in tuberculosis patients in Indonesia. Int J Tuberc Lung Dis 2002;6:497-502.

8. Mehta J, Shantaveerapa H, Byrd R, Morton S, et al. Utility of rifampin blood levels in the treatment and follow-up of active pulmonary tuberculosis in patients who were slow to respond to routine directly observed therapy. Chest 2001;120:1520-4.

9. Weiner M, Burman W, Vernon A, et al. Low isoniazid concentrations and outcome of tuberculosis treatment with once-weekly isoniazid and rifapentine. Am J Respir Crit Care Med 2003; 167:1341-7.

10. Weiner M, Benator BW, Peloquin CA, et al. Association between acquired rifamycin resistance and the pharmacokinetics of rifabutin and isoniazid among patients with HIV and tuberculosis. Clin Infect Dis 2005;40:1481-91. therapy. This was the case despite following detailed guidelines regarding rifabutin dosing in such patients suggested by online resources such as those provided by the Centers for Disease Control and Prevention (USA). We conclude that TDM is potentially useful in the treatment of active TB, but is currently underused - reflecting the very limited access to appropriate laboratory facilities.

ACKNOWLEDGEMENTS: The authors thank Dr Chuck Charles Peloquin for providing additional information and background literature, the staff of the TB clinic and medical records department in the Montreal Chest Institute for their assistance in completing the study, and Mme Ria Choe for assistance in manuscript preparation. Dr Babalik conducted the primary data gathering and data analysis, and drafted the first version of the manuscript. Dr Menzies conceived and designed the study, assisted in data analysis and made final revisions to the manuscript. Dr Mannix and $\mathrm{Mr}$ Francis assisted in data gathering and made critical revisions to the manuscript.

CONFLICTS OF INTEREST: None of the authors have any conflicts of interests, financially or otherwise, with the content of this article.

FUNDING: Dr Babalik was supported by a fellowship from the European Union. Dr Menzies received a Chercheur National salary support award from the Fonds de la Recherche en Santé du Quebec.

11. Department of Health and Human Services/Centers for Disease Control and Prevention. Managing Drug Interactions in the Treatment of HIV-Related Tuberculosis, 2007.

$<$ http://www.cdc.gov/tb/TB_HIV_Drugs/default.htm> (Accessed on July 4, 2011).

12. Boulanger C, Hollender E, Farrell K, et al. Pharmacokinetic evaluation of rifabutin in combination with lopinavir-ritonavir in patients with HIV infection and active tuberculosis. Clin Infect Dis 2009;49:1305-11.

13. Holdiness MR. Clinical pharmacokinetics of the antituberculosis drugs. Clin Pharmacokinet 1984;9:511-44.

14. Dawson-Saunders B, Trapp RG. Basic and clinical biostatistics. Norwalk: Appleton \& Lange, 1990.

15. Chideya S, Winston C, Peloquin CA, Bradford WZ, et al. Isoniazid, rifampin, ethambutol, and pyrazinamide pharmacokinetics and treatment outcomes among a predominantly HIV-infected cohort of adults with tuberculosis from Botswana. Clin Infect Dis 2009; 48:1685-94.

16. Ray J, Gardiner I, Marriott D. Managing antituberculosis drug therapy by therapeutic drug monitoring of rifampicin and isoniazid. Internal Med J 2003;33:229-34.

17. Sahai J, Gallicano K, Swick L, et al. Reduced plasma concentrations of antituberculosis drugs in patients with HIV infection. Ann Intern Med 1997;127:289.

18. Holland DP, Hamilton CD, Weintrob AC, et al. Therapeutic drug monitoring of antimycobacterial drugs in patients with both tuberculosis and advanced human immunodeficiency virus infection. Pharmacotherapy 2009;29:503-10.

19. Menzies D, Lewis M, Oxlade O. Costs for tuberculosis care in Canada. Can J Publ Health 2008;99:391-6.

20. Mitnick CD, McGee B, Peloquin CA. Tuberculosis pharmacotherapy: Strategies to optimize patient care. Expert Opin Pharmacother 2009;10:381-401. 


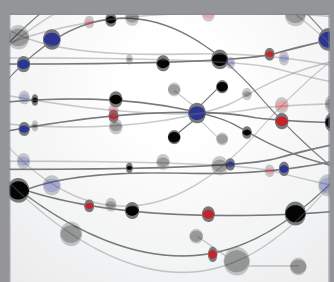

The Scientific World Journal
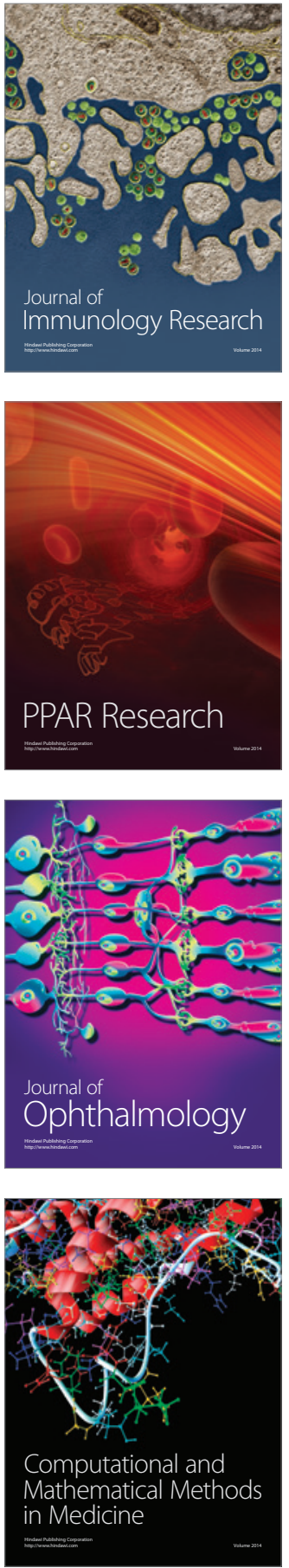

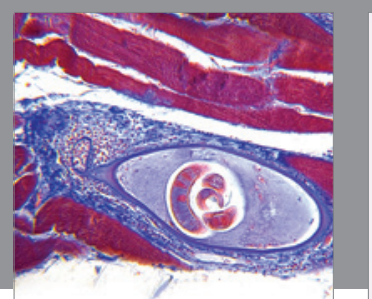

Gastroenterology Research and Practice

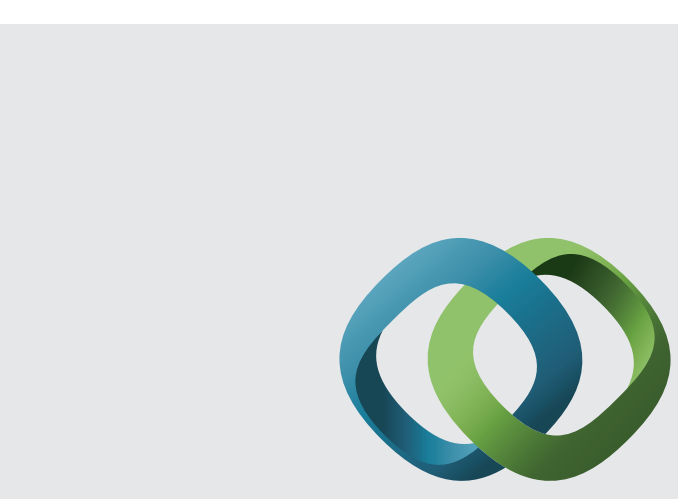

\section{Hindawi}

Submit your manuscripts at

http://www.hindawi.com
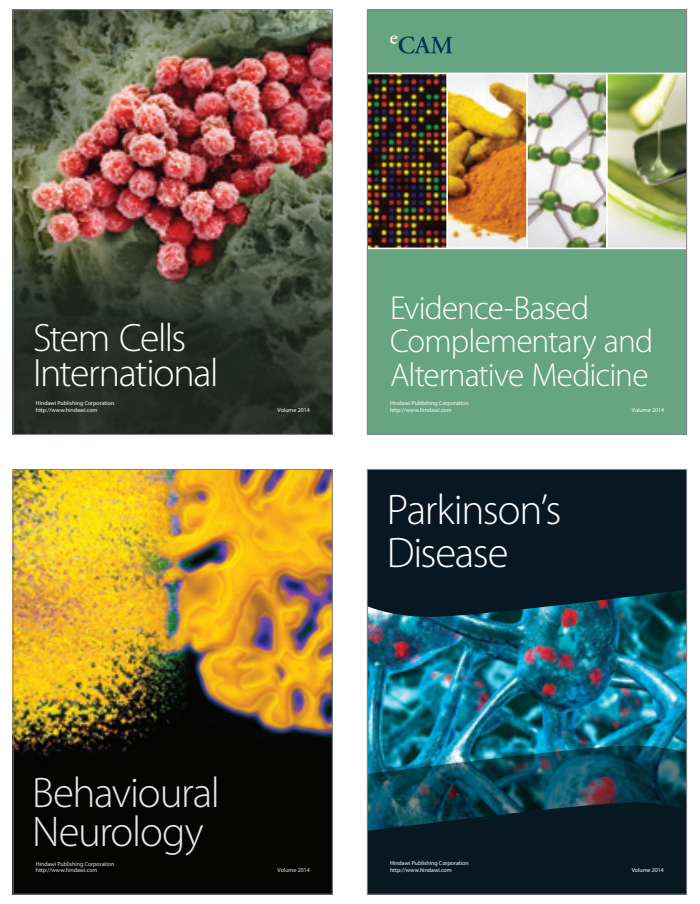
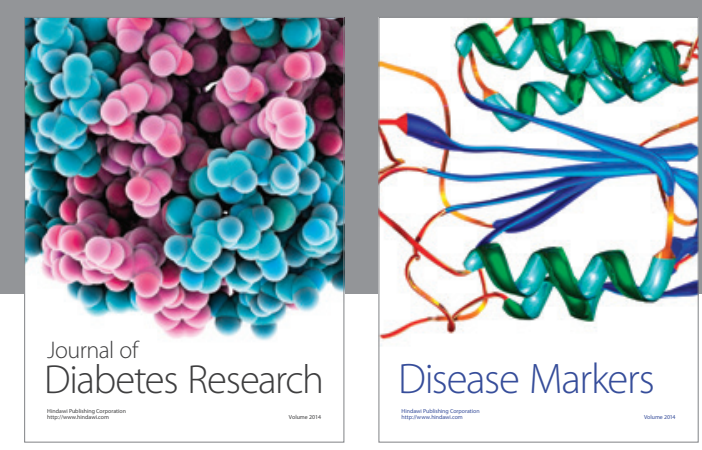

Disease Markers
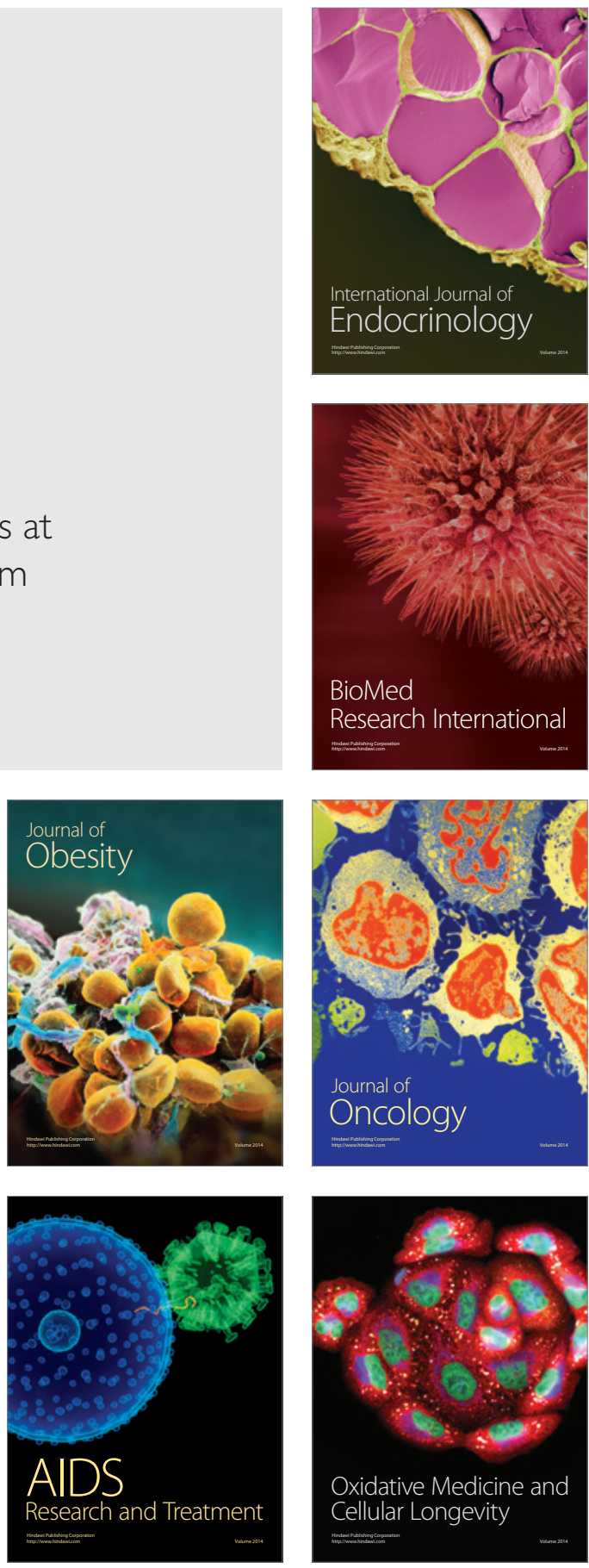